\title{
$B$ cells in central nervous system disease: diversity, locations and pathophysiology
}

Rajiv W. Jain and V. Wee Yong ${ }^{凶}$

Abstract $\mid$ B cells represent a relatively minor cell population within both the healthy and diseased central nervous system (CNS), yet they can have profound effects. This is emphasized in multiple sclerosis, in which B cell-depleting therapies are arguably the most efficacious treatment for the condition. In this Review, we discuss how B cells enter and persist in the CNS and how, in many neurological conditions, B cells concentrate within CNS barriers but are rarely found in the parenchyma. We highlight how B cells can contribute to CNS pathology through antibody secretion, antigen presentation and secretion of neurotoxic molecules, using examples from CNS tumours, CNS infections and autoimmune conditions such as neuromyelitis optica and, in particular, multiple sclerosis. Overall, understanding common and divergent principles of B cell accumulation and their effects within the CNS could offer new insights into treating these devastating neurological conditions.

\section{B1 B cells}

A subset of mature B cells with limited B cell receptor diversity predominantly found within the peritoneal and pleural cavities that typically differentiates directly into plasma cells, forming few memory cells

Follicular B cells A subset of mature B cells, also known as B2 B cells, that represents the majority of $B$ cells in the body and are commonly found within all lymphoid organs.

Marginal zone B cells A subset of mature $B$ cells with limited B cell receptor diversity that is predominantly found in the marginal zone of the spleen and which typically differentiates directly into plasma cells but can also generate memory cells.

Hotchkiss Brain Institute and the Department of Clinical Neurosciences, University of Calgary, Calgary, Alberta, Canada.

凶e-mail:vyong@ucalgary.ca https://doi.org/10.1038/ s41577-021-00652-6
B cells were originally identified through studies searching for the cellular source of antibodies, which led to the discovery of antibody-secreting plasma cells and, eventually, their precursors, the B cells. Similarly, the study of B cells in the context of central nervous system (CNS) disorders has often started as a result of antibody detection in cerebrospinal fluid (CSF) or at sites of injury. The first indication that B cells could contribute to multiple sclerosis (MS) was the detection of antibodies in CSF ${ }^{1}$. The study of the antigenic targets of these antibodies led to the discovery of the autoimmune diseases neuromyelitis optica (NMO) and myelin oligodendrocyte glycoprotein (MOG) antibody-associated disorder and their designations as separate entities from MS. Many other autoimmune encephalitides have been discovered, such as anti $N$-methyl-D-aspartate receptor (NMDAR) encephalitis, wherein antibodies target various neuronal proteins and contribute to these disorders ${ }^{1}$. Antibodies may also influence neurodegenerative processes in Parkinson disease and Alzheimer disease ${ }^{1}$ as well as in CNS infections ${ }^{2}$.

The notion that B cells can contribute to CNS pathology independently of antibody production was first hinted at in MS, where the depletion of CD20+ B cells was shown to be an effective treatment ${ }^{3}$. This was an unexpected result as B cells represent a minor population of immune cells in the CNS during MS and, as we will discuss in this review, are not appreciably found in the CNS parenchyma at sites of damage but are instead found behind barriers of entry into the CNS. Since this discovery, the study of how B cells contribute to CNS immunity and pathology independently of antibody production and from a distance are a growing field of study. There are still many unanswered questions regarding whether $\mathrm{B}$ cells contribute to these disorders from within or outside the CNS, which B cell subsets are responsible for promoting pathology and repair, and what mechanisms B cells use to influence CNS pathology. In this Review, we will focus specifically on the role of $\mathrm{B}$ cells within the CNS, covering their representation and localization in health and CNS disorders. We review how $B$ cells enter and persist in the CNS and what effector functions they use to promote pathology with a particular focus on MS as the vast amount of relevant data has come from the MS field. These discussions emphasize $\mathrm{B}$ cells as important regulators of CNS responses in both homeostasis and disease.

\section{A refresher on $B$ cells}

For a thorough refresher on B cell immunology, we suggest reading the reviews cited here and in FIC. 1 as we will only cover basic B cell immunology. Mature $\mathrm{B}$ cells are those that have completed development. They are broadly separated into antigen-experienced or antigen-inexperienced (naive) B cells. Naive B cells can be further differentiated into B1 B cells, follicular B cells (also known as B2 B cells) and marginal zone B cells. Naive $B$ cells are recruited to participate in immune responses through B cell receptor (BCR) stimulation initiated by antigen binding in combination with co-stimulatory 
Germinal centre B cells

This subset of activated B cells is an intermediate stage of differentiation and is the precursor of higher-affinity memory B cells and plasma cells.

Memory B cells A subset of $B$ cells that is antigen experienced and has reacquired a quiescent phenotype and can participate in secondary immune responses, or sometimes refers to activated B cells that retain an activated phenotype.

T-bet ${ }^{+}$memory B cells A subset of memory $B$ cells that expresses the transcription factor T-bet whose numbers tend to increase with age as well as in autoimmune disorders and viral infections.

Plasma cells

Terminally differentiated B cells that produce large amounts of antibodies and are short-lived unless they find a survival niche allowing long-term maintenance. signalling or in combination with $\mathrm{T}$ cell help to induce a $\mathrm{T}$ cell-dependent immune response $\mathrm{e}^{4}$ (FIG. 1). The products of $\mathrm{T}$ cell-dependent immune responses are differentiated B cell subsets such as memory B cells, T-bet ${ }^{+}$memory $B$ cells and plasma cells.

B cells contribute to immunity through antibody production, antigen presentation and production of secreted products. When antibodies bind their targets, they can initiate antibody-dependent cellular cytotoxicity, complement-dependent cytotoxicity or phagocytosis ${ }^{5}$. Antigen presentation is a process wherein $B$ cells endocytose antigens, then process and load them onto MHC class II molecules to present to CD4 ${ }^{+}$ $\mathrm{T}$ cells. Antigen presentation by B cells is essential in maintaining germinal centres and to activate and polarize $\mathrm{CD}^{+} \mathrm{T}$ cell responses. Another major mechanism employed by B cells to affect immune responses is the secretion of pro-inflammatory and anti-inflammatory cytokines. Regulatory $B\left(B_{\text {reg }}\right)$ cells generally produce anti-inflammatory cytokines while antigenexperienced B cells generally secrete pro-inflammatory cytokines $^{3}$.

\section{Where are B cells in the healthy CNS?}

Before immune cells can enter the CNS parenchyma, they must first pass through restrictive barriers of the post-capillary venules (blood-brain barrier (BBB)), meninges (blood-meningeal barrier) and choroid plexus (blood-CSF barrier). Here, we provide a brief description of how these barriers interact with immune cells; for more in-depth descriptions see REFS ${ }^{6,7}$.

Post-capillary venules in the CNS are surrounded by a thick extracellular matrix with barrier properties maintained by endothelial cells, pericytes, astrocytes, microglia and neurons; this barrier retains immune cells around the blood vessel in areas known as VirchowRobin spaces ${ }^{8}$. The meninges is separated into the dura mater that is in contact with the skull, arachnoid mater and the innermost pia mater that overlies the parenchyma ${ }^{7}$. Blood vessels are present in the subarachnoid space of the meninges where immune cells can migrate across these blood vessels into the subarachnoid space and CSF. Directly associated with the pia mater, astrocyte end-feet maintain the glial limitans, a barrier that prevents immune cell entry into the parenchyma. During inflammation, immune cells may be able to cross the pia mater to enter the CNS parenchyma although this process is incompletely described. Immune cells entering the choroid plexus first extravasate across capillaries to enter the choroid plexus stroma; they then cross the choroid plexus epithelial cell barrier to enter the $\mathrm{CSF}^{6}$.

In mice, B cells are rarely found in the healthy CNS parenchyma and are only found in small numbers in CSF, the choroid plexus and the subdural meninges. However, B cells are constitutively present in the dural meninges and represent $\sim 15-30 \%$ of the total CD $45^{\text {hi }}$ cells in this location ${ }^{9-11}$. The vast majority of B cells in

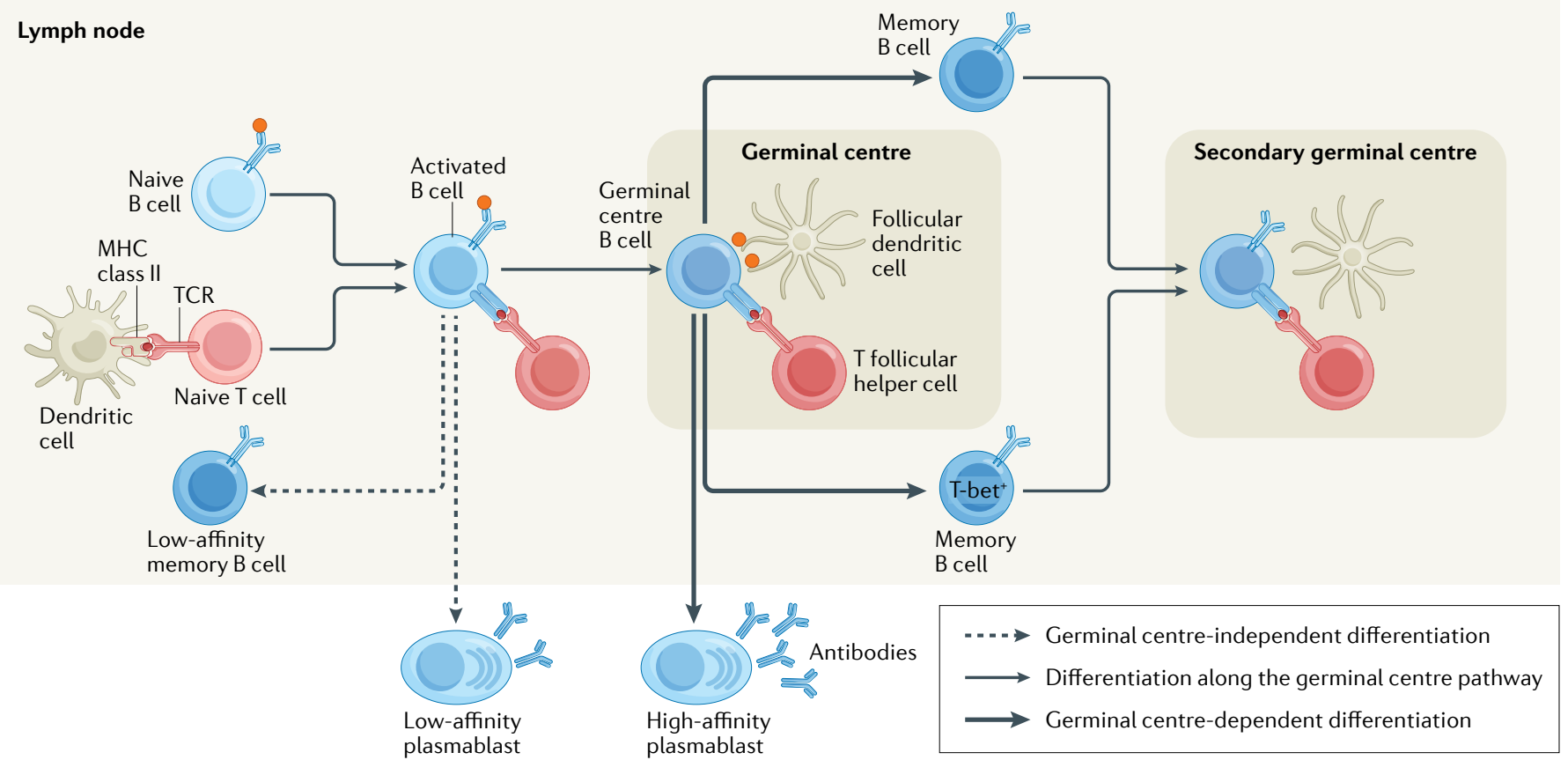

Fig. 1 | T cell-dependent B cell differentiation. T cell-dependent B cell differentiation is initiated when $B$ cells engage with their antigen through their $B$ cell receptor (BCR) and a cognate T cell response is activated (left side of figure). Activated B cells and T cells then move to the border of the B cell follicle and $T$ cell zones by modifying their expression of chemokine receptors ${ }^{119}$ and form cognate pairs through physical interactions. These interactions can induce $B$ cell class-switch recombination to change their initial lgD and $\lg M$ isotypes to $\lg \mathrm{G}, \lg \mathrm{A}$ or $\lg \mathrm{E}^{120}$. These interactions drive the differentiation of mostly $\lg \mathrm{M}^{+}$ low-affinity memory B cells, low-affinity plasmablasts, and pre-germinal centre
B cells that re-enter the B cell follicle with $T$ follicular helper cells to seed a new germinal centre 4 . The germinal centre is composed of germinal centre B cells, T follicular helper cells and supportive cells, such as follicular dendritic cells, that organize and maintain the germinal centre environment ${ }^{4}$. In the germinal centre, T follicular helper cells induce somatic hypermutation in germinal centre B cells and direct them to differentiate into high-affinity short-lived and high-affinity long-lived plasma cells and into T-bet ${ }^{-}$or T-bet $^{+}$ memory B cells ${ }^{4,12}$. Memory B cells can then later participate in an immune response if they are re-exposed to antigen (secondary germinal centre). 
Regulatory B ( $\left.\mathrm{B}_{\mathrm{ree}}\right)$ cells This subset of $B$ cells suppresses inflammation through the secretion of anti-inflammatory proteins or through physical interactions.

Plasmablasts

The precursor to plasma cells that has begun to produce antibodies but also retains some features of B cells, such as surface $B$ cell receptor and MHC class II expression, and is still proliferating. the dura meninges are B2 B cells and a large fraction of these are immature B cells generated in the skull's bone marrow $^{10,11}$. Most mature CNS B cells are naive $\operatorname{IgM}^{+}$ cells ${ }^{10}$ with unmutated BCRs although there are some $\mathrm{IgA}^{+} \mathrm{B}$ cells ${ }^{11}$. Furthermore, $\mathrm{B}$ cells in the CNS are mainly tissue resident cells that were generated locally ${ }^{10,11}$. The capacity of peripheral B cells to take up residence in the healthy CNS is limited in young mice but increases as mice age and this increase is primarily due to age-associated B cells - whose phenotype overlaps with T-bet ${ }^{+} \mathrm{B}$ cells ${ }^{12}$ - accumulating in the brain ${ }^{10}$. In young animals Ig $\mathrm{A}^{+}$plasma cells dominate the meninges ${ }^{13}$ but, as mice age, $\mathrm{IgG}^{+}$and $\mathrm{IgM}^{+}$plasma cells become more common $^{10}$. Of note, the population of IgA $\mathrm{A}^{+}$plasma cells found in the healthy CNS is partially derived from $\operatorname{Ig} \mathrm{A}^{+}$ plasma cells that are generated in the gut ${ }^{13}$.

In humans, B cells and plasma cells are rarely found in the parenchyma of the healthy CNS although small numbers are reported ${ }^{14,15}$. They are present in low numbers in perivascular spaces ${ }^{14-16}$ and more frequently found in the meninges ${ }^{16}$, particularly in the dura mater ${ }^{11,13}$. B cells can be detected in healthy CSF in humans; however, few plasma cells are found, corresponding with the low levels of antibody seen in $\mathrm{CSF}^{17,18}$.

\section{B cells in neurological conditions}

$\mathrm{B}$ cells can be recruited to the CNS as a result of CNS infection ${ }^{13}$ and even due to sleep disruption ${ }^{19}$. Indeed, in many neurological conditions, increased levels of IgM, IgA and IgG are seen in $\mathrm{CSF}^{17}$. Here, we review B cells in the context of CNS cancers, infections of the CNS and autoimmune disorders affecting the CNS finally focusing on MS. The locations of B cells and plasma cells in these disorders are summarized in Supplementary Table 1.

Cancers of the CNS. Glioblastoma is a deadly tumour that arises within the CNS. The immune cell composition of glioblastoma predominantly consists of microglia and macrophages, while B cells and T cells are present in small numbers in these tumours. By flow cytometry, $0.66 \%$ of the immune cells found in glioblastoma are $\mathrm{B}$ cells ${ }^{20}$. In meningiomas, which are tumours that form in the meninges, $\mathrm{B}$ cell frequencies are highly variable but, on average, they represent $0.03 \%$ of all cells in the tumour $^{21}$. Cancers of the CNS also include those that have metastasized from extra-cranial locations. Patients with B cell lymphomas can have metastasis to the $\mathrm{CNS}^{22}$, which present as solid tumours of B cells retained within the meninges or perivascular spaces or as diffuse tumours that spread through the parenchyma ${ }^{22}$. Whether B cells promote or suppress tumour growth likely depends on the types of B cells in the tumour. Glioblastoma promotes the conversion of B cells into $\mathrm{B}_{\text {reg }}$ cells that sustains tumorigenicity ${ }^{23}$. However, in the right inflammatory environment, B cells can elicit an anti-glioblastoma immune response ${ }^{24}$.

CNS infections. Many patients infected with coronavirus disease 2019 (COVID-19) develop signs of neurological dysfunction with evidence of pathology ongoing in the $\mathrm{CNS}^{25}$. Single-cell sequencing-based studies have either found no evidence of B cell and plasma cell expansion in $\mathrm{CSF}^{26}$ or have reported expansion ${ }^{27}$ of these populations in patients with neurological manifestations of COVID-19.

CNS pathology induced by coronaviruses is not unique to COVID-19, as many other coronaviruses, including the original severe acute respiratory syndrome virus and Middle East respiratory syndrome ${ }^{28}$ virus, drive pathology within the CNS. In mouse models of CNS coronavirus infection, peripheral plasmablasts, memory B cells and naive B cells ${ }^{29}$ are recruited into the CNS. Plasma cells enter the parenchyma, where they can contribute to localized antibody production that is associated with protection from infection ${ }^{30}$, whereas naive $\mathrm{B}$ cells stay within the perivascular and meningeal compartments ${ }^{31}$. Thus, the mouse models of CNS coronavirus infection support a protective role of $\mathrm{B}$ cells entering the CNS.

Evidence of $\mathrm{B}$ cells playing a protective role from within the CNS is also seen in other CNS-trophic viral and bacterial infections. In these conditions, virus-specific or bacteria-specific antibodies are produced within CSF and the parenchyma of infected CNS tissue where antibodies are presumed to contribute to viral and/or bacterial clearance ${ }^{2,32,33}$.

Autoimmune diseases. B cells have a major role in contributing to immune responses through their capacity to secrete antibodies. However, the secretion of antibodies can be detrimental in certain circumstances as exemplified by the autoimmune CNS disorders NMO (which is associated with anti-aquaporin 4 antibodies), antiNMDAR encephalitis (associated with anti-NMDAR antibodies) and MOG antibody-associated disorder ${ }^{1}$. B cells and plasma cells within the CNS may also contribute to neuropsychiatric forms of systemic lupus erythematosus based on studies in animal model $s^{34}$ and also on the finding that oligoclonal bands of immunoglobulin are detected in the CSF of $26.5 \%$ of patients with systemic lupus erythematosus who have neuropsychiatric manifestations ${ }^{35}$.

There is evidence of antibody production from clonally expanded plasma cells within the CSF of patients with NMO or with anti-NMDAR encephalitis ${ }^{36,37}$. Nonetheless, only patients with anti-NMDAR encephalitis show a concentration of pathogenic antibodies within the CNS and, in patients with MOG antibody-associated disorder and patients with NMO, most autoreactive antibodies are produced peripherally ${ }^{1,36}$. Indeed, unlike in patients with MS, where oligoclonal bands of immunoglobulin are consistently detected in CSF, only $15-30 \%$ of patients with NMO have oligoclonal bands of immunoglobulin in CSF, suggesting that plasma cell expansion in the CSF of patients with NMO is not robust ${ }^{1}$.

\section{B cells in MS}

MS is an inflammatory and degenerative disease of the CNS where oligodendrocytes and neurons are lost in white and grey matter leading to the accumulation of neurological deficits. The progression of MS is broadly separated into a relapsing-remitting MS (RRMS) phase, in which neurological deficits manifest and recede, and a phase of secondary progressive MS (SPMS; if 


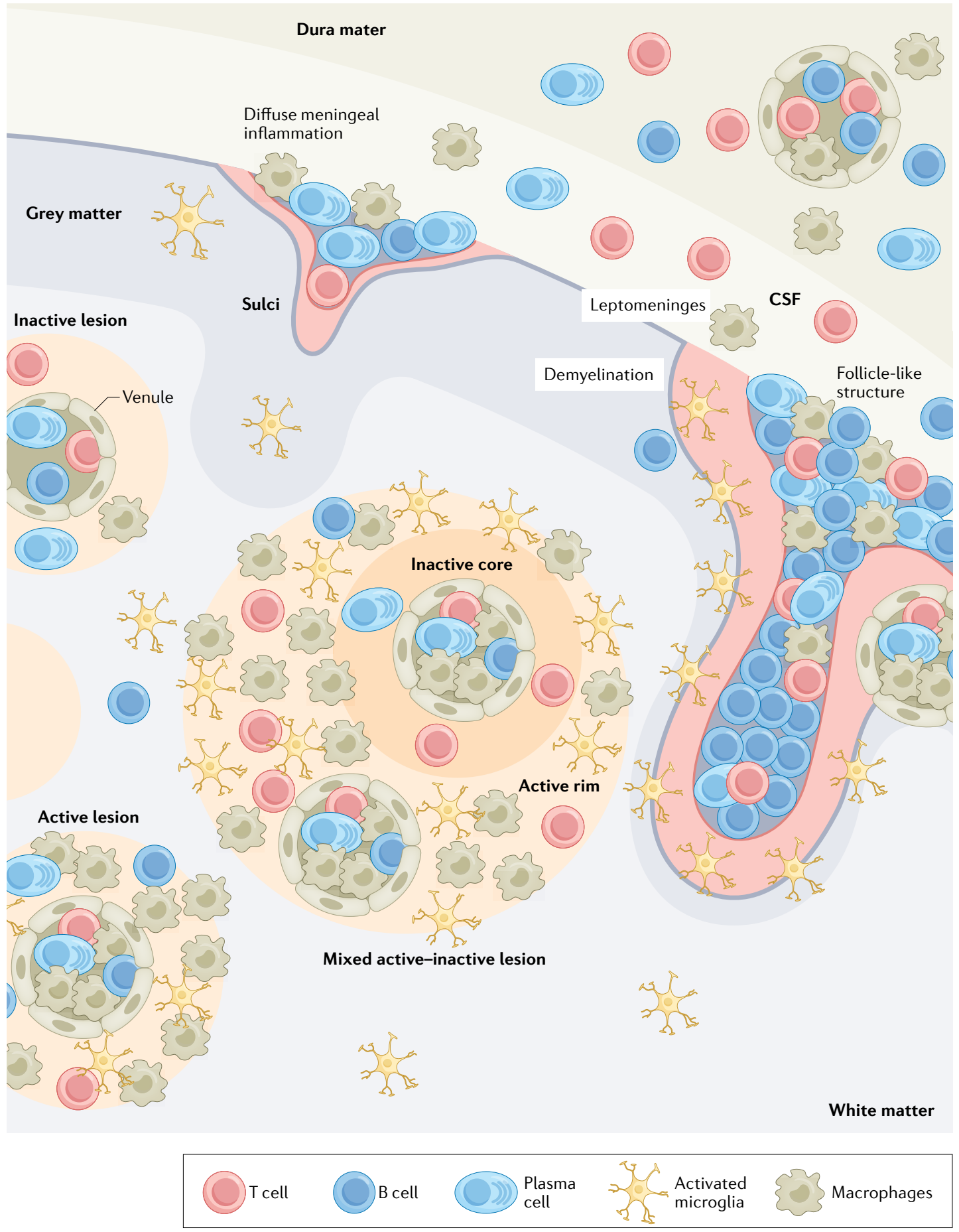

progression follows RRMS) or primary progressive MS (PPMS), wherein neurological disability accumulates with minimal periods of recovery. Monoclonal antibodies that deplete $\mathrm{CD} 20^{+} \mathrm{B}$ cells are highly effective in treating the relapsing phase of MS and, in some patients, also the progressive forms of $\mathrm{MS}^{3}$. Although B cells can contribute to MS pathology from the periphery ${ }^{38}$, there is considerable evidence that B cells enter CNS barrier regions to participate in CNS pathology.

Locations of B cells in the CNS of patients with MS. MS lesions can be broadly segregated into grey matter lesions, where the cortical locations are often associated with meningeal inflammation, and white matter lesions. The latter can be active lesions filled with immune cells, mixed active-inactive lesions that have an actively demyelinating rim associated with macrophage/microglia and a demyelinated immunologically inactive centre, or inactive demyelinated lesions (FIG. 2). Active and mixed active-inactive lesions can be further subdivided into demyelinating and post-demyelinating based on whether myelin proteins are present or absent within macrophage/microglia, respectively. We encourage the reader to follow the recent consensus ${ }^{39}$ on the nomenclature of MS lesions that addresses the ambiguities of old naming conventions present in older papers. 
4 Fig. 2 | Locations of B cells in multiple sclerosis lesions. Depictions of lesions are approximations of the average number of $B$ cells relative to $T$ cells and macrophage/ microglia in the meninges and in grey and white matter lesions. B cell and plasma cell populations are expanded in the cerebrospinal fluid (CSF) of patients with multiple sclerosis but only represent $0.5-11 \%$ and $0.25-12 \%$ of total immune cells, respectively ${ }^{32,44,48,121}$. Grey matter lesions can be split into those that have diffuse B cell infiltration or the formation of large collections of B cells in B cell aggregates in the meningeal space that typically form at the bottom of sulci $i^{16,40-42,52}$. Relative to diffuse $B$ cells in the meningeal space, B cell aggregates are associated with increased infiltration of macrophages, T cells and some plasma cells into the central nervous system parenchyma, grey matter demyelination and proximal microglial activation ${ }^{41,42,52,54,55,58}$. In white matter lesions, B cells and plasma cells are commonly found in the perivascular spaces of postcapillary venules but are rarely found in the parenchyma ${ }^{15}$. Plasma cells are found in the same locations as B cells but are more likely to enter the parenchyma of the central nervous system than $B$ cells regardless of the lesion type ${ }^{14,15}$.

In patients with MS, B cells and plasma cells in the CNS are primarily found in the meninges and perivascular locations but they are also present in the parenchyma in small numbers ${ }^{40-42}$ (FIG. 2). There is preliminary evidence of $\mathrm{B}$ cells entering the choroid plexus in $\mathrm{MS}^{43}$ but this requires confirmation. B cells and plasma cells are also expanded in the CSF from patients with MS relative to that from healthy humans ${ }^{44,45}$, especially during periods of active disease where class-switched B cells and plasma cells clonally expand ${ }^{46}$. B cells are also 1-3 times more prevalent in the CSF of patients with RRMS relative to those with progressive $\mathrm{MS}^{47,48}$.

Based on post-mortem studies, meningeal and perivascular B cell aggregates are highly variable in MS, where they can be nearly absent in the lesions of some patients or dominate the lesions of others ${ }^{15}$. Meningeal and perivascular B cells are more numerous in RRMS and SPMS relative to PPMS, whereas plasma cells are more prevalent in progressive over RRMS ${ }^{14,15}$. B cells are more common in the parenchyma and perivascular spaces of active lesions relative to mixed active-inactive or inactive lesions whereas plasma cells are common in mixed active-inactive or inactive lesions ${ }^{14,15,49,50}$. Both B cells and plasma cells are rare in normal-appearing white matter $^{14,49,50}$.

Cortical lesions associated with meningeal inflammation often have B cells that largely remain in the meninges ${ }^{15}$. In one study using high resolution MRI analysis of patients with MS, meningeal inflammation was found in $\sim 33 \%$ of patients with progressive MS and in $\sim 19 \%$ of patients with RRMS ${ }^{51}$, although the specific percentages can vary between studies. Meningeal B cell inflammation can be diffuse or extensive, the latter often associated with large 'follicle-like' structures ${ }^{42}$. These structures are referred to as follicle-like due to their resemblance to B cell follicles in secondary lymphoid organs, and they are characterized by separate zones of $\mathrm{B}$ cells and $\mathrm{T}$ cells and by large numbers of associated plasma cells ${ }^{42,52}$ (FIG. 2). As the resemblance of these structures to follicles remains controversial, we will refer to them as B cell aggregates. Based on post-mortem histology studies, the frequency of B cell aggregates in SPMS is estimated to be $\sim 40 \%^{16,40,52}$. Similar studies in PPMS found that $30 \%$ of PPMS have B cell aggregates although these meningeal aggregates do not achieve the same levels of organization seen in SPMS ${ }^{41,52}$. A similar frequency of B cell aggregates between MS subtypes is found in spinal cords of patients with MS although B cell aggregates are less common than in the brain ${ }^{42}$. Generally, patients that have one B cell aggregate in their brain are more likely to have additional B cell aggregates throughout their $\mathrm{CNS}^{42,52}$.

$B$ cell invasion of the CNS in patients with MS or in animal models of experimental autoimmune encephalomyelitis (EAE) has been studied in sufficient detail to provide an overview of the specific B cell subsets encountered in the CNS (Supplementary Table 2).

Association of B cells with MS lesions. An important question is whether the B cells in the CNS of patients with MS are contributing to disease directly or whether they are simply bystanders attracted to the CNS by the inflammatory environment. Suggesting an active role, patients that have a CSF immune cell repertoire biased towards B cells and plasma cells over other immune cell types show faster disease progression ${ }^{53}$. When comparing cortical lesions that do or do not have B cell aggregates in post-mortem samples, individuals with $B$ cell aggregates are more likely to have transitioned earlier to being wheelchair-bound and to have died earlier in both PPMS and SPMS ${ }^{16,40,41,52,54}$. In a study of SPMS autopsies with or without $\mathrm{B}$ cell aggregates in the meninges, there is a strong correlation between the presence of B cell aggregates and cortical demyelination ${ }^{54}$. When meningeal inflammation is not segregated by the presence of B cell aggregates in patients with SPMS, there is an inconsistent and weak correlation between the degree of meningeal immune cell infiltration and cortical demyelination, suggesting that $\mathrm{B}$ cell aggregates are the primary locations driving cortical demyelination ${ }^{41,52}$. Meningeal B cell aggregates may also be associated with demyelination in adjacent white matter ${ }^{42,49,55,56}$; however, this relationship has not been found in all studies ${ }^{52,54}$.

$B$ cell aggregates are also associated with pronounced local neuronal damage ${ }^{57}$. Biopsies taken from patients during early MS or following clinically isolated syndrome (which is the first manifestation of a demyelinating event) suggest that meningeal inflammation, either diffuse or concentrated in density in the meninges, is associated with demyelination in the cortex but that neuronal loss is only seen underneath dense aggregates in the meninges of subpial lesions ${ }^{57,58}$. Similar results have also been seen in post-mortem studies comparing patients with SPMS with or without $B$ cell aggregates ${ }^{41,52}$ and this damage can even extend to the spinal cord ${ }^{42}$. Neuronal loss typically occurs in a gradient from the upper layers of the cortex and progresses inwards ${ }^{54}$ and is associated with apoptotic markers in neurons ${ }^{58}$. Indeed, the presence of B cell aggregates in the meninges is associated with faster cortical thinning ${ }^{54}$. This gradient of damage emanating away from the meningeal $\mathrm{B}$ cell aggregates suggests that $\mathrm{B}$ cells are secreting factors that diffuse into the surrounding CNS parenchyma and either directly damage CNS cells or may indirectly injure them by promoting inflammatory polarization of microglia as iNOS ${ }^{+} \mathrm{TNF}^{+}$phagocytes are found in proximity to $\mathrm{B}$ cell aggregates ${ }^{54}$. Nonetheless, while prominent meningeal inflammation is associated with the upregulation of inflammatory cytokines locally and within CSF, 
Thelper $17\left(T_{H} 17\right)$ cells

A subset of $C D 4^{+} T$ cells

characterized by the

expression of the transcription

factor ROR $\mathrm{T} T$ and the

expression of IL-17A that is

commonly associated with

immunity against extracellular

pathogens. it is also associated with increased expression of IL-10 in both $\mathrm{MS}^{59}$ and $\mathrm{EAE}^{60}$. Thus, while $\mathrm{B}$ cell aggregates are associated with worse pathology, some components may also inhibit inflammation.

$B$ cell aggregates may also affect the recruitment of other cell types into the parenchyma of the CNS. The parenchyma and small vessels in the cortex of areas associated with B cell aggregates are seen to have more $\mathrm{CD}^{+} \mathrm{T}$ cells, $\mathrm{B}$ cells, macrophages and plasma cells, particularly in the upper layers of the cortex ${ }^{16,54,55}$ (FIG. 2). $\mathrm{CD}^{+} \mathrm{T}$ cells and macrophages also accumulate in the meninges associated with $\mathrm{B}$ cell aggregates ${ }^{16,55}$.

Although the relevance of B cells in white matter inflammation is less well researched, one study found that the absence of $\mathrm{B}$ cells from white matter lesions is associated with slower and less severe disease progression, reduced $\mathrm{T}$ cell infiltration into lesions, lower incidence of mixed active-inactive lesions, and reduced incidence and intensity of oligoclonal bands ${ }^{50}$. Furthermore, another study that separated white matter lesions by the presence or absence of IgM or IgG deposits found that there is more $\mathrm{B}$ cell and plasma cell infiltration into white matter lesions when immunoglobulins are present, particularly for lesions containing IgG deposits ${ }^{49}$. Indeed, IgG deposition in lesions is associated with increased B cell and plasma cell infiltration into perivascular spaces, the parenchyma of the lesion, and increased $B$ cell influx into the meninges near the lesion. Thus, B cells and plasma cells in white matter lesions contribute to MS pathology.

\section{Recruitment and survival in the CNS}

Much of what is known of B cell recruitment into the CNS comes from the MS literature. B cells can be recruited through the $\mathrm{BBB}$, meningeal barriers and choroid plexus. Each of these sites differs with respect to structure but the overall process of moving from blood into these locations proceeds similarly (FIG. 3). Below, we will outline the mechanisms B cells use to pass through these barriers. Of note, $T$ helper $17\left(T_{H} 17\right)$ cells play an important role in opening the $\mathrm{BBB}$ and creating an environment for B cells to persist in the CNS, adding another layer of complexity to CNS B cell recruitment and persistence beyond what is discussed here ${ }^{61}$.

Rolling stage of B cell recruitment. Mice with genetic deletion of L-selectin (also known as CD62L), used by $\mathrm{B}$ cells to enter lymph nodes, show no deficiency of B cell infiltration into the CNS in the EAE model ${ }^{62}$. Indeed, B cells in the CNS of animals with EAE have low levels of L-selectin expression ${ }^{63}$ and the ligands for L-selectin are not well represented in EAE or MS lesions ${ }^{64}$; this suggests that L-selectin is not important for B cell recruitment into the CNS. One factor known to affect the rolling stage is the adhesion molecule ALCAM, which is upregulated on activated B cell subsets in $\mathrm{MS}^{65}$. ALCAM-deficient B cells roll at a faster rate on activated endothelial cells in vitro and ALCAM-blocking antibodies reduce disease severity and B cell recruitment into the CNS in mice with $\mathrm{EAE}^{65}$. Nonetheless, deletion of this single molecule does not completely prevent rolling on the endothelium, suggesting that other molecules affect the rolling stage of B cell recruitment.

Chemokines associated with B cell entry. Several chemokines associated with B cell migration have been studied in MS and EAE, including CXC-chemokine ligand 10 (CXCL10), CXCL12, CXCL13, CC-chemokine ligand 19 (CCL19) and CCL21. Each of these is known to affect specific B cell subsets and have different patterns of expression in MS lesions (Supplementary Table 3). Three other chemokines associated with B cell entry

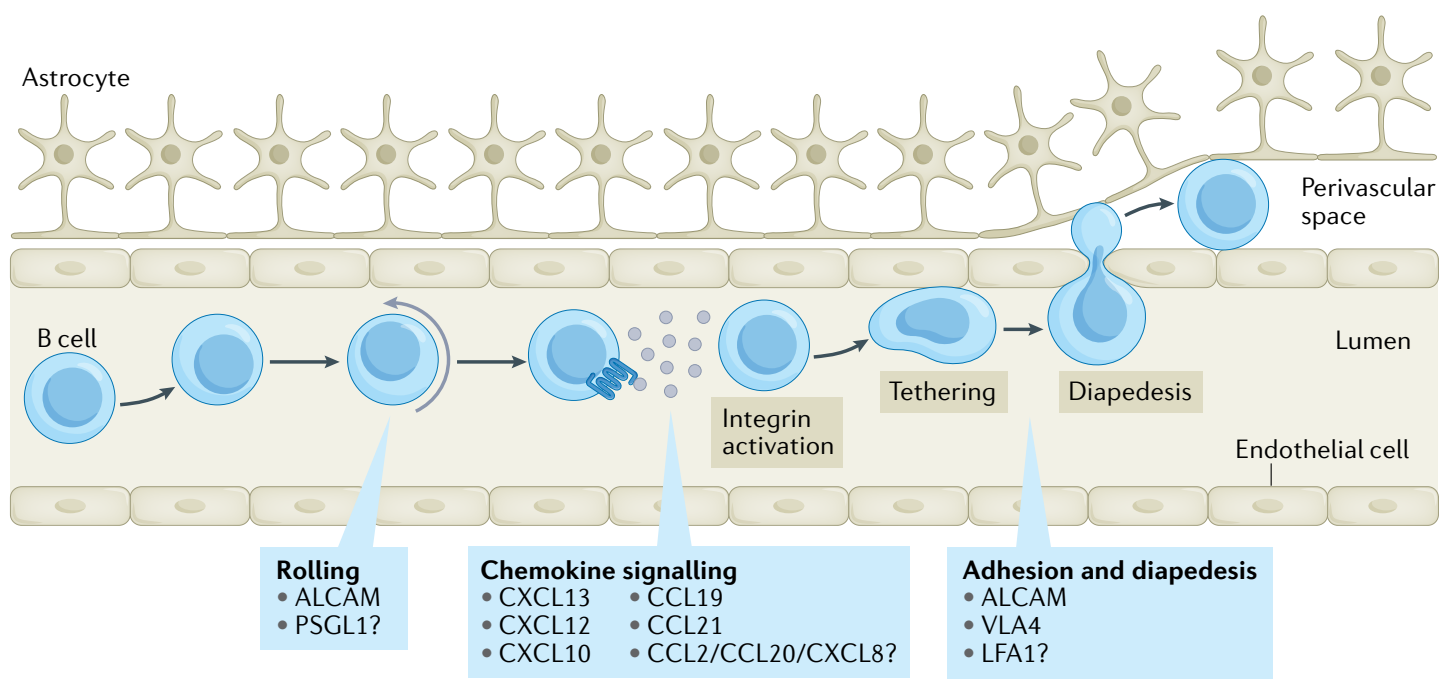

Fig. 3 | Mechanisms of B cell recruitment into the CNS. Immune cells are recruited into the central nervous system (CNS) by first rolling along the lumen of the blood vessel and being activated by locally produced cytokines. Activation of B cells, including by chemokines found on endothelial cells, then induces conformational changes in integrins, allowing high affinity interactions and firm tethering to the vessel; this enables cells to migrate across the endothelial cell layer into CNS barriers such as the perivascular space ${ }^{6,7}$. Although this process is not fully understood in the context of B cell entry into the CNS, several molecules shown in the boxes are known to affect their migration into the CNS at distinct stages whereas other molecules remain contentious or inadequately studied. CXCL, CXC-chemokine ligand; CCL, CC-chemokine ligand. 
T follicular helper cells A subset of $\mathrm{CD}^{+} \mathrm{T}$ cells characterized by the expression of the transcriptional repressor Bcl6 and the chemokine receptor CXCR5 allowing these cells to co-localize with $B$ cells and promote their differentiation through physical interactions into the CNS based on in vitro studies are CCL20, IL-8 (also known as CXCL8) and CCL2 (REFS ${ }^{66,67}$ ); however, in vivo evidence of a role for these chemokines is lacking in MS or EAE. Additionally, CC-chemokine receptor 5 (CCR5) is overexpressed on CSF B cells in $\mathrm{MS}^{46}$ but it is unknown whether this affects $B$ cell migration into the CNS.

Integrins and cell adhesion molecules. B cells express several integrins and cell adhesion molecules that facilitate their entry into the CNS. ALCAM promotes B cell diapedesis across brain endothelial cells ${ }^{65}$. Other important molecules are the integrin LFA1 and its cell adhesion molecule ligand ICAM1, both of which are upregulated on activated B cells in $\mathrm{MS}^{65}$. Blocking ICAM1 reduces human $\mathrm{B}$ cell migration across brain endothelial cell layers in culture ${ }^{65,66}$ but there is no in vivo evidence supporting this.

The best characterized molecule affecting B cell trafficking into the CNS is VLA4. This integrin is expressed highly on activated B cells in EAE and MS ${ }^{65,68}$ and is needed for B cells to cross brain endothelial cells in vitro ${ }^{65,66}$. In mouse models, deletion of VLA4 on $B$ cells reduces $B$ cell recruitment into the $\mathrm{CNS}^{69,70}$ and leads to reduction of EAE severity in B cell-dependent $\mathrm{EAE}^{70}$. Nonetheless, the effects of VLA4 blockade on EAE severity could be due to inhibition of peripheral $\mathrm{B}$ cell activation ${ }^{69}$. Blocking VLA4 in patients with MS is known to affect memory B cell trafficking ${ }^{3}$ leading to the concentration of memory B cells in blood corresponding with therapeutic benefit ${ }^{68}$. In contrast, treating patients with MS with anti-VLA4 antibodies led to no changes in B cell and plasma cell numbers in CSF, and biopsies of white matter lesions showed slight elevations in plasma cell density ${ }^{71}$. Overall, the mouse data strongly supports the idea that VLA4 affects B cell recruitment into the CNS whereas the human data suggests that some but not all B cell types are affected.

Antigen-specificity in recruitment. Activated T cells, regardless of their antigen specificity, are recruited to the CNS; however, only the T cells that encounter their antigen in the CNS are retained at this site in large numbers over time ${ }^{72}$. In the context of inflammation, B cells are recruited to the CNS in an antigen-independent manner that does not appear to follow the same rules as $\mathrm{T}$ cell recruitment ${ }^{72}$. Similarly, plasma cells specific for ovalbumin protein, rotavirus and non-self-antigens can be recruited into the CNS during $\mathrm{EAE}^{73,74}$ and can even persist as long-lived cells ${ }^{73}$. Thus, specificity for an antigen within the CNS is not explicitly required for $\mathrm{B}$ cells to take up residence in the CNS.

B cell survival in the CNS. The primary factors that influence B cell survival are BAFF and APRIL, both of which are available in MS lesions (Supplementary Table 3). Mature B cells are dependent on BAFF and, as they commit to plasma cell differentiation, they become increasingly more dependent on APRIL ${ }^{75,76}$. In contrast, memory B cells are much less dependent on survival factors relative to other B cell subsets ${ }^{76}$. Beyond BAFF and APRIL, astrocytes make undefined survival factors that promote the survival of B cells ${ }^{77}$. Plasma cell survival is enhanced by fibronectin and hypoxic conditions ${ }^{75}$, which can be found in MS lesions.

Another important factor that influences B cell and plasmablast survival in the CNS is their interaction with T cells. Both activated B cells and plasmablasts are susceptible to cell death but can be rescued by $\mathrm{CD}^{+} \mathrm{T}$ cell help ${ }^{78}$. These interactions are likely to occur given that autoreactive $\mathrm{T}$ cell clones derived from the CNS of patients with MS induce the proliferation of autoreactive B cells, suggesting that they form stable interactions $^{38}$. Furthermore, invasion of the CNS by $\mathrm{T}$ follicular helper cells in EAE is associated with $\mathrm{B}$ cell population expansion in the $\mathrm{CNS}^{79}$. Thus, $\mathrm{B}$ cell-T cell interactions are likely important in maintaining $\mathrm{B}$ cells in the CNS.

$B$ cell recruitment and survival in other CNS diseases. CXC-chemokine receptor 3 (CXCR3) expression by plasma cells is associated with plasma cell entry into the CNS in $\mathrm{NMO}^{80}$ and viral encephalomyelitis ${ }^{30}$. Additionally, the CXCR3 ligands, CXCL10 and CXCL9, are upregulated in the CNS during viral encephalomyelitis ${ }^{30}$. In B cell lymphoma and in the healthy CNS, CXCL12 is expressed highly on the blood vessel endothelium, resulting in B cell recruitment into perivascular spaces ${ }^{81}$. However, CXCL12 also promotes egress from perivascular spaces unless countered by astrocyte-derived CCL19, which results in $B$ cell accumulation in perivascular spaces and entrance to the parenchyma. Notably, the same study showed that increased expression of VCAM1 and ICAM1 on the blood endothelium in itself is not sufficient for $\mathrm{B}$ cell retention in the CNS. Many B cell lymphomas also express IL-15 that can induce the expression of CXCR3 and PSGL1, a selectin that can influence the rolling stage of recruitment, further promoting CNS recuitment ${ }^{82}$.

Despite high expression of the $B$ cell survival factor receptors BAFF-R, BCMA and TACI by B cell lymphomas, there is no increase in BAFF expression in the CNS during CNS lymphoma relative to healthy CNS tissue ${ }^{83}$. By contrast, in a mouse model of CNS viral infection, there is evidence of increased BAFF and APRIL expression within the $\mathrm{CNS}^{84}$. Thus, in combination with the data from the MS literature, inflammation in the CNS is likely needed to induce increased expression of these survival factors.

\section{B cell functions in MS lesions}

$B$ cells as direct mediators of damage. Antibodies contribute to the pathology of MS lesions and in animal models through several different mechanisms, including opsonization, initiation of complement deposition, facilitating $\mathrm{B}$ cell and $\mathrm{T}$ cell activation, and antibodydependent cellular cytotoxicity (FIG. 4). Of note, there is currently no consistently identified autoantigen target for antibodies in $\mathrm{MS}^{85}$ although antibodies are seen in MS lesions.

Some patients with MS have what are referred to as 'pattern II lesions', which have antibody and complement deposits in white matter ${ }^{86}$, providing the potential of formation of a membrane attack complex that can be 
Thelper $1\left(T_{H} 1\right)$ cells

A subset of $C D 4^{+} T$ cells characterized by the

expression of the transcription factor T-bet and the expression of IFN $\gamma$ that is commonly associated with cellular immunity. destructive (FIG. 4). Individual lesions within the same patient can differ, with some lesions having IgM or IgG deposits but not others ${ }^{87}$. Most active, mixed activeinactive and inactive lesions have either IgM or IgG deposits, with IgG being more common ${ }^{87}$. Various antibody isotypes are detected in MS lesions and each has typical deposition patterns. IgG and IgM are found in lesions in proximity to demyelinated axons, degrading myelin and myelin debris, dying cells, and within macrophages ${ }^{49,87,88}$. Both IgM and IgG have been associated with oligodendrocytes in lesions, normal appearing white matter, and in newly forming lesions ${ }^{87,88}$. IgA is reported in lesions typically bound to axons and vasculature $^{89}$. However, it remains unclear whether the pathology of these lesions is driven by CNS-derived or peripherally produced antibodies as therapeutic plasma exchange that removes circulating serum antibodies benefits these subjects ${ }^{90}$. In favour of CNS-produced antibodies contributing to antibody deposition, antibodies from the CSF of patients with RRMS or with progressive MS bind human cerebellum sections ${ }^{91}$. Similar results have been produced using cloned antibodies derived from single B cells, plasma cells or antibodies from $\mathrm{CSF}^{92}$ albeit not in all studies ${ }^{33,94}$. Thus, the question of whether antibodies are pathogenic in MS is equivocal.

Another possibility that has been highlighted more recently is that $B$ cells may be contributing to MS in a non-conventional manner: through the production of pathogenic microvesicles or exosomes. The evidence for this comes from results showing that large secreted
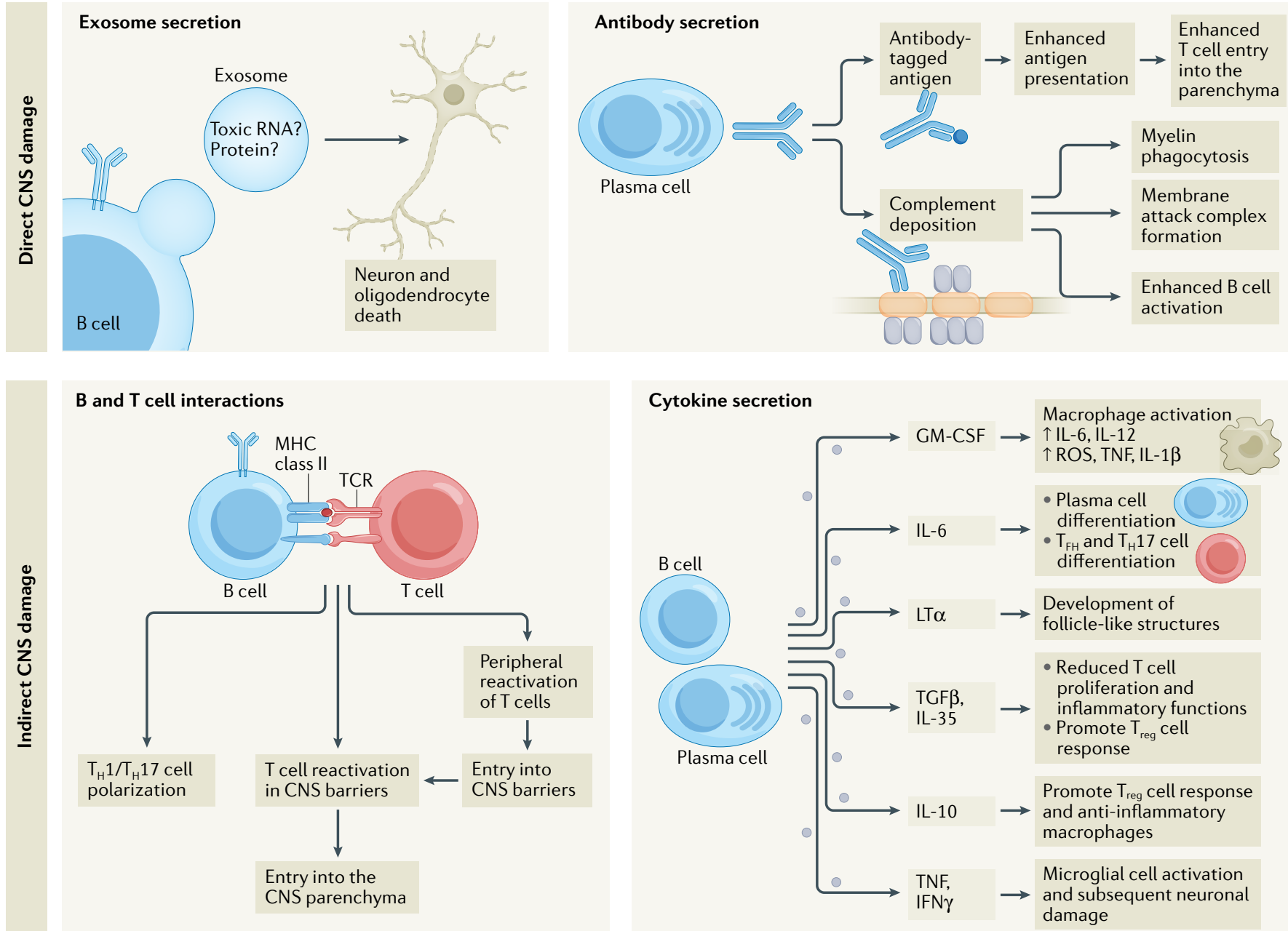

Fig. 4 | Direct and indirect mechanisms that B cells use to affect multiple sclerosis pathology. B cells can directly damage the central nervous system (CNS) using secreted molecules, including exosomes that induce death in oligodendrocytes and neurons, through unknown mechanisms (top left panel). Antibodies directly contribute to multiple sclerosis pathology by facilitating $\mathrm{T}$ cell reactivation in CNS barriers, promoting their migration into the parenchyma ${ }^{122}$, and by promoting the deposition of complement on myelin ${ }^{86}$ to enhance myelin phagocytosis ${ }^{88,123}$, membrane attack complex formation on myelin ${ }^{86}$, and the enhanced activation of B cell immune responses ${ }^{124}$ (top right panel). B cells can indirectly affect CNS pathology by inducing the differentiation of autoreactive Thelper $1\left(T_{H} 1\right)$ cell and $T_{H} 17$ cell responses in the periphery and within the CNS, in part through CD80 and CD86

upregulation (bottom left panel). However, B cells can also inhibit T cell responses through expression of PDL1 (not depicted). B cell-derived secreted cytokines also affect CNS pathology (bottom right panel): GM-CSF promotes macrophage-driven pathology ${ }^{102}$; IL-6 enhances plasma cell differentiation and survival, $\mathrm{T}$ follicular helper $\left(\mathrm{T}_{\mathrm{FH}}\right)$ cell differentiation, and $\mathrm{T}_{\mathrm{H}} 17$ polarization ${ }^{125,126}$. Lymphotoxin- $\alpha(L T \alpha)$ is important for organizing large clusters of $B$ cells in the meninges and for the formation of 'follicle-like' structures ${ }^{61}$. TNF and IFN $\gamma$ are associated with microglial cell activation and neuron and oligodendrocyte death ${ }^{105-107}$. TGF $\beta 1$ and IL-35 reduce T cell proliferation and inflammatory T cell polarization and promote regulatory $T\left(T_{\text {reg }}\right)$ cell responses ${ }^{112,114}$. IL-10 affects T cells similarly ${ }^{111,117}$ but also alters macrophage polarization and may promote remyelination ${ }^{127}$. ROS, reactive oxygen species. 
particles from B cells induce death in cultured oligodendrocytes and neurons ${ }^{95,96}$, which was attributed to secreted exosomes ${ }^{95}$ (FIG. 4). Currently, there is no obvious mechanism by which these exosomes are inducing cell death in their targets or evidence to show whether exosomes are produced by B cells in the CNS in vivo.

B cells indirectly promote CNS damage. In patients with MS and in animals with EAE, it is likely that B cells in the CNS can promote injury indirectly by supporting $T$ cell responses through physical interactions ${ }^{15,38,42,63}$ given the close association of $\mathrm{T}$ cells and $\mathrm{B}$ cells in perivascular and meningeal compartments (FIG. 4). In EAE, $\mathrm{B}$ cells in both the periphery and the CNS upregulate the co-stimulatory molecule CD80, suggesting that they are primed for interaction with $\mathrm{T}$ cells ${ }^{63,97}$. Indeed, autoantigen-driven $\mathrm{B}$ cell-T cell interactions influence the incidence and induction of relapses, timing of disease onset, and chronicity of $\mathrm{EAE}^{79,97-99}$. These interactions primarily influence $\mathrm{T}_{\mathrm{H}} 1$ cell and/or $\mathrm{T}_{\mathrm{H}} 17$ cell polarization of $\mathrm{CD} 4{ }^{+} \mathrm{T}$ cells and induce waves of $\mathrm{T}$ cell infiltration into the CNS from the periphery and within the $\mathrm{CNS}^{79,97,98,100}$.

The above is consistent with a recent study showing that memory B cells induce autoreactive T cell expansion in patients with MS during remission periods, ultimately activating these $\mathrm{T}$ cells to enter the brain and reactivating them again within the $\mathrm{CNS}^{38}$. Inflammatory memory B cell subsets that highly express the co-stimulatory molecules CD80 and CD86 concentrate within the CSF of patients with MS, suggesting that B cells in the CNS are primed to interact with $\mathrm{T}$ cells ${ }^{44,68,101}$. This is partially explained by inflammatory astrocytes producing soluble factors that promote the expression of CD86 on B cells ${ }^{77}$. Overall, there is considerable evidence for a pathogenic role for B cell and $\mathrm{T}$ cell interactions in MS and EAE.

Another emerging and important role for B cells in MS is their capacity to make pro-inflammatory cytokines that can modify the inflammatory environment to activate cells such as macrophages and/or microglia and $\mathrm{T}$ cells. There is evidence of peripheral B cells expressing several inflammatory cytokines in MS, including GM-CSF, TNF, IL-6 and lymphotoxin- $\alpha^{102-104}$; CSF $B$ cells are known to make lymphotoxin- $\alpha$ and TNF $^{103,104}$ although data on GM-CSF and IL- 6 are not available. These cytokines can modulate CNS damage and the immune response through several mechanisms summarized in FIG. 4.

Of note, TNF is expressed highly in cortical lesions associated with IFN $\gamma^{59}$, particularly in those with B cell aggregates $^{105}$. Bulk RNA sequencing of meningeal $B$ cell aggregate-associated grey matter lesions suggest that an imbalance of TNF-mediated death signalling occurs through increased expression of TNFR1 (which induces cell death) over that of TNFR2 (which induces survival pathways) ${ }^{106}$. In EAE, forced expression or injection in the subarachnoid space of TNF and IFN $\gamma$ leads to enhanced EAE induction characterized by a gradient of microglial cell activation and neuronal loss and associated with demyelination emanating away from cortical lesions ${ }^{105,107}$ reminiscent of MS. Temporally, TNF and IFN $\gamma$ induce an initial expansion of inflammatory microglia that directly associate and phagocytose pre-synapse proteins ${ }^{108}$. Later, the microglial numbers contract to the density of healthy controls and microglia lose some of their inflammatory polarization, become more ramified and are associated with increased neuronal loss. Similar observations were made analysing cortical lesions of MS patients ${ }^{108}$. Of note, T-bet ${ }^{+}$memory B cells can produce IFN $\gamma^{12}$ and, thus, $\mathrm{B}$ cells could be self-sufficient in driving TNF-mediated and IFN $\gamma$-mediated CNS pathology.

The damage dealt by pro-inflammatory cytokines may be balanced by regulatory cytokines produced by $\mathrm{B}_{\text {reg }}$ cells though several mechanisms (FIG. 4). Regulatory roles for B cells are exemplified in B cell-deficient or $B$ cell-depleted mice that develop worse EAE than mice with full B cell repertoires ${ }^{109,110}$. The anti-inflammatory cytokines IL-10 (REFS ${ }^{74,110,111}$ ), TGF $\beta 1$ (REFS ${ }^{112,113}$ ) and IL-35 (REF. ${ }^{114}$ ) have all been found to be made by subsets of $\mathrm{B}_{\text {reg }}$ cells in either EAE or MS. Additionally, $\mathrm{B}_{\text {reg }}$ cells can also inhibit $T$ cell responses through physical interactions using PD-L1 (REF. ${ }^{115}$ ). Although $\mathrm{B}_{\text {reg }}$ cells modulate EAE, it is not clear whether these cells act in the periphery or within the CNS. In support of $B_{\text {reg }}$ cells working within the CNS, IL- $10^{+}$plasma cells are seen in active/mixed active-inactive MS lesions ${ }^{15}$ and IL-10 is inducible in some B cells from EAE spinal cords ${ }^{116}$. Moreover, in one EAE study, $30 \%$ of B cells in meningeal B cell aggregates express mRNA for either IL-10 or IL-35 $\left(R_{E F}{ }^{60}\right)$. In contrast, others have found that large numbers of IL-10-producing plasmablasts are seen in lymph nodes that drain the site of immunization in EAE but IL-10-expressing B-lineage cells are not seen in the $\mathrm{CNS}$ of $\mathrm{EAE}^{111,117}$. Furthermore, preventing B cells from accessing these lymph nodes abolished the capacity of $\mathrm{B}_{\text {reg }}$ cells to suppress $\mathrm{EAE}^{117}$. One population that clearly enters the CNS in MS and EAE are IL- $10^{+}$gut-derived $\operatorname{IgA}^{+}$plasma cells that suppress EAE severity ${ }^{18,74}$. Thus, there is a clear role for regulatory B cells in MS but whether the primary site of inhibition is within the CNS or in the periphery remains unanswered.

\section{B cell functions in other CNS disorders}

The entry of B cell lymphomas into the CNS is sometimes associated with local damage but, more often, it is not associated with any damage, suggesting that the presence of B cells in the CNS, even in large numbers, is not necessarily enough to induce pathology $y^{22}$. Even when B cell lymphomas are associated with damage, it is likely that a prior insult to the CNS led to the upregulation of chemokines that promote $\mathrm{B}$ cell recruitment rather than the $\mathrm{B}$ cells being directly pathogenic ${ }^{22,81}$. Thus, B cell entry into the CNS is insufficient to induce CNS pathology, suggesting that a trigger is needed to convert B cells to an injurious state.

The most commonly studied mechanism for B cells to induce CNS damage is through the production of autoantibodies. An example of this is seen in NMO, where anti-AQP4 antibodies induce astrocyte death and destruction of CNS barriers by removing astrocyte endfeet $^{1}$. Antibody deposition leads to complement deposition, antibody-dependent cellular cytotoxicity and enhanced T cell immunity. In MOG antibody-associated 
disorder, antibodies induce demyelination emanating away from blood vessels in affected tissues ${ }^{1}$. In lesions, antibodies are associated with complement deposition, complement-dependent cytotoxicity, macrophages containing myelin and $\mathrm{T}$ cells. In contrast to these more inflammatory disorders, anti-NMDAR encephalomyelitis is driven by IgG4 antibodies that are not associated with complement deposition or antibody-dependent cellular cytotoxicity but, instead, it is likely that these antibodies affect the crosslinking and internalization of $\mathrm{NMDAR}^{1}$. Thus, inflammation is not always required for $\mathrm{B}$ cells to be pathogenic. Autoantibody-driven pathology is also seen in patients with COVID-19. While virus-specific antibodies and BCRs are detected among plasma cells and B cells in CSF, many of these cells are also reactive against CNS antigens ${ }^{27}$. Thus, in addition to autoimmune diseases, viral infection can trigger secondary autoimmunity in the CNS.

\section{Gaps of knowledge and new directions}

We have discussed the diversity of B cells found in the brain in both health and disease as well as where to locate them. It is clear that B cells are present in the CNS in many disorders and that they affect disease outcomes. However, there are still many gaps in our understanding of precisely how these B cells contribute to disease and where B cells and plasma cells are located in the CNS during these conditions. Even in MS, where B cells have been studied in detail, there are remaining uncertainties regarding the localization of specific subsets of B cells in CNS lesions or whether particular B cell subsets exist in the CNS. Precisely which mechanisms B cells use to mediate CNS outcomes also remain unclear. Answers to these questions are needed to define which subsets of $\mathrm{B}$ cells should be targeted to overcome CNS pathology and which subsets confer benefits in the CNS.

In the context of MS, several mechanisms have been suggested to explain how B cells contribute to MS. Here, we have highlighted that B cells interact with $\mathrm{T}$ cells to promote $\mathrm{T}$ cell pathology and that several types of secreted B cell products, including cytokines, extracellular vesicles and antibodies, may all contribute to MS pathology. What is not clear is the degree to which these various mechanisms are active within the CNS versus the periphery and which subsets of B cells primarily use these disease mechanisms to promote pathology. It has also been noted that, while B cell infiltration into the CNS in MS is associated with CNS damage, this is not universally true of all CNS conditions ${ }^{22}$. Currently, it is not clear if there is a specific trigger that converts B cells into pathogenic entities or whether their diverse roles are simply due to the chronicity of conditions or other factors. It is also clear that the gut microbiome can regulate $\mathrm{B}$ cell functions in the $\mathrm{CNS}^{118}$ but this is poorly defined. A better understanding of how B cells differ in the various conditions discussed herein would be of great benefit to understand the mechanisms that B cells use to promote neurodegeneration or neuroprotection and could potentially unlock needed therapeutic targets.

Published online 13 December 2021
1. Sabatino, J. J. Jr., Probstel, A. K. \& Zamvil, S. S $B$ cells in autoimmune and neurodegenerative central nervous system diseases. Nat. Rev. Neurosci. 20, 728-745 (2019)

2. Gilden, D. H. Infectious causes of multiple sclerosis. Lancet Neurol. 4, 195-202 (2005).

3. Li, R., Patterson, K. R. \& Bar-Or, A. Reassessing B cell contributions in multiple sclerosis. Nat. Immunol. 19, 696-707 (2018)

4. Akkaya, M., Kwak, K. \& Pierce, S. K. B cell memory: building two walls of protection against pathogens. Nat. Rev. Immunol. 20, 229-238 (2020).

5. Kang, T. H. \& Jung, S. T. Boosting therapeutic potency of antibodies by taming Fc domain functions. Exp. Mol. Med. 51, 1-9 (2019).

6. Korn, T. \& Kallies, A. T cell responses in the central nervous system. Nat. Rev. Immunol. 17, 179-194 (2017).

7. Rua, R. \& McGavern, D. B. Advances in meningeal immunity. Trends Mol. Med. 24, 542-559 (2018).

8. Agrawal, S. M. et al. Extracellular matrix metalloproteinase inducer shows active perivascular cuffs in multiple sclerosis. Brain 136, 1760-1777 (2013).

9. Korin, B. et al. High-dimensional, single-cell characterization of the brain's immune compartment. Nat. Neurosci. 20, 1300-1309 (2017). This is one of the few studies to precisely quantify the number of immune cells in the healthy CNS across the parenchyma, meninges and choroid plexus.

10. Brioschi, S. et al. Heterogeneity of meningeal B cells reveals a lymphopoietic niche at the CNS borders. Science 373, eabf9277 (2021).

11. Schafflick, D. et al. Single-cell profiling of CNS border compartment leukocytes reveals that B cells and their progenitors reside in non-diseased meninges. Nat. Neurosci. 24, 1225-1234 (2021).

12. Rubtsova, K., Rubtsov, A. V., Cancro, M. P. \& Marrack, P. Age-associated B cells: a T-bet-dependent effector with roles in protective and pathogenic immunity. J. Immunol. 195, 1933-1937 (2015).
13. Fitzpatrick, Z. et al. Gut-educated IgA plasma cells defend the meningeal venous sinuses. Nature 587 , 472-476 (2020).

This publication highlights how $B$ and plasma cells accumulate in the dura mater in health and disease and identified the gut as a source of IgA plasma cells.

14. Frischer J. M et al. The relation between inflammation and neurodegeneration in multiple sclerosis brains. Brain 132, 1175-1189 (2009).

15. Machado-Santos, J. et al. The compartmentalized inflammatory response in the multiple sclerosis brain is composed of tissue-resident $\mathrm{CD} 8^{+} \mathrm{T}$ lymphocytes and B cells. Brain 141, 2066-2082 (2018). This paper has the most thorough quantification of B and plasma cells in MS white matter lesions.

16. Howell, $\mathrm{O}$. W. et al. Meningeal inflammation is widespread and linked to cortical pathology in multiple sclerosis. Brain 134, 2755-2771 (2011).

17. Prasad, R. Immunoglobulin levels in serum and cerebrospinal fluid in certain viral infections of the central nervous system. J. Infect. Dis. 148, 607 (1983).

18. Probstel, A. K. et al. Gut microbiota-specific lgA ${ }^{+}$ $B$ cells traffic to the CNS in active multiple sclerosis. Sci. Immunol. 5, eabc7191 (2020).

19. Korin, B. et al. Short-term sleep deprivation in mice induces B cell migration to the brain compartment. Sleep 43, zsz222 (2020).

20. Kmiecik, J. et al. Elevated $\mathrm{CD}^{+}$and $\mathrm{CD} 8^{+}$tumorinfiltrating immune cells correlate with prolonged survival in glioblastoma patients despite integrated immunosuppressive mechanisms in the tumor microenvironment and at the systemic level. J. Neuroimmunol. 264, 71-83 (2013).

21. Domingues, P. H. et al. Immunophenotypic identification and characterization of tumor cells and infiltrating cell populations in meningiomas. Am. J. Pathol. 181, 1749-1761 (2012)

22. Kuhlmann, T., Lassmann, H. \& Bruck, W. Diagnosis of inflammatory demyelination in biopsy specimens: a practical approach. Acta Neuropathol. 115 275-287 (2008)
23. Han, S. et al. Glioma cell-derived placental growth factor induces regulatory B cells. Int. J. Biochem. Cell Biol. 57, 63-68 (2014).

24. Lee-Chang, $\mathrm{C}$. et al. Activation of $4-1 \mathrm{BBL}^{+} \mathrm{B}$ cells with CD40 agonism and IFNgamma elicits potent immunity against glioblastoma. J. Exp. Med. 218, e20200913 (2021).

25. Matschke, J. et al. Neuropathology of patients with COVID-19 in Germany: a post-mortem case series. Lancet Neurol. 19, 919-929 (2020).

26. Heming, M. et al. Neurological manifestations of COVID-19 feature T cell exhaustion and dedifferentiated monocytes in cerebrospinal fluid. Immunity 54, 164-175.e6 (2021).

27. Song, E. et al. Divergent and self-reactive immune responses in the CNS of COVID-19 patients with neurological symptoms. Cell Rep. Med. 2, 100288 (2021).

28. Khateb, M., Bosak, N. \& Muqary, M. Coronaviruses and central nervous system manifestations. Front. Neurol. 11, 715 (2020).

29. Phares, T. W., DiSano, K. D., Stohlman, S. A. ¿ Bergmann, C. C. Progression from IgD $\mathrm{IgM}^{+}$ to isotype-switched B cells is site specific during coronavirus-induced encephalomyelitis. J. Virol. 88 8853-8867 (2014).

30. Tschen, S. I. et al. CNS viral infection diverts homing of antibody-secreting cells from lymphoid organs to the CNS. Eur. J. Immunol. 36, 603-612 (2006)

31. DiSano, K. D., Stohlman, S. A. \& Bergmann, C. C. Activated $\mathrm{GL} 7^{+} \mathrm{B}$ cells are maintained within the inflamed CNS in the absence of follicle formation during viral encephalomyelitis. Brain Behav. Immun. 60, 71-83 (2017)

32. Cepok, S. et al. Short-lived plasma blasts are the main $B$ cell effector subset during the course of multiple sclerosis. Brain 128, 1667-1676 (2005).

33. Kowarik, M. C. et al. CXCL13 is the major determinant for $\mathrm{B}$ cell recruitment to the CSF during neuroinflammation. J. Neuroinflammation 9, 93 (2012). 
34. Stock, A. D. et al. Tertiary lymphoid structures in the choroid plexus in neuropsychiatric lupus. $\mathrm{JCl}$ Insight 4 e124203 (2019).

35. Mok, M. Y., Chan, E. Y., Wong, W. S. \& Lau, C. S Intrathecal immunoglobulin production in patients with systemic lupus erythematosus with neuropsychiatric manifestations. Ann. Rheum. Dis. 66 846-847 (2007)

36. Kowarik, M. C. et al. The cerebrospinal fluid immunoglobulin transcriptome and proteome in neuromyelitis optica reveals central nervous systemspecific B cell populations. J. Neuroinflammation 12 19 (2015).

37. Malviya, M. et al. NMDAR encephalitis: passive transfer from man to mouse by a recombinant antibody. Ann. Clin. Transl. Neurol. 4, 768-783 (2017).

38. Jelcic, I. et al. Memory B cells activate brain-homing, autoreactive $\mathrm{CD}^{+} \mathrm{T}$ cells in multiple sclerosis. Cell 175, 85-100.e23 (2018) In this study, memory B cells from MS patients are shown to induce $\mathrm{CD4}^{+} \mathrm{T}$ cell proliferation and induce these cells to enter the CNS

39. Kuhlmann, T. et al. An updated histological classification system for multiple sclerosis lesions. Acta Neuropathol. 133, 13-24 (2017).

40. Bell, L., Lenhart, A., Rosenwald, A., Monoranu, C. M. $\Sigma$ Berberich-Siebelt, F. Lymphoid aggregates in the CNS of progressive multiple sclerosis patients lack regulatory T cells. Front. Immunol. 10, 3090 (2019).

41. Choi, S. R. et al. Meningeal inflammation plays a role in the pathology of primary progressive multiple sclerosis. Brain 135, 2925-2937 (2012).

42. Reali, C. et al. B cell rich meningeal inflammation associates with increased spinal cord pathology in multiple sclerosis. Brain Pathol. 30, 779-793 (2020).

43. Monaco, S., Nicholas, R., Reynolds, R. \& Magliozzi, R. Intrathecal inflammation in progressive multiple sclerosis. Int. J. Mol. Sci. 21, 8217 (2020).

44. Corcione, A. et al. Recapitulation of B cell differentiation in the central nervous system of patients with multiple sclerosis. Proc. Natl Acad. Sci. USA 101, 11064-11069 (2004).

45. Schafflick, D. et al. Integrated single cell analysis of blood and cerebrospinal fluid leukocytes in multiple sclerosis. Nat. Commun. 11, 247 (2020).

46. Ramesh, A. et al. A pathogenic and clonally expanded $B$ cell transcriptome in active multiple sclerosis. Proc. Natl Acad. Sci. USA 117, 22932-22943 (2020).

47. Di Pauli, F. et al. Features of intrathecal immunoglobulins in patients with multiple sclerosis. J. Neurol. Sci. 288, 147-150 (2010).

48. Eggers, E. L. et al. Clonal relationships of CSF B cells in treatment-naive multiple sclerosis patients. $\mathrm{JCl}$ Insight 2, e92724 (2017).

49. Munoz, U. et al. Main role of antibodies in demyelination and axonal damage in multiple sclerosis. Cell Mol. Neurobiol. https://doi.org/10.1007/ s10571-021-01059-6 (2021).

50. Fransen, N. L. et al. Absence of B cells in brainstem and white matter lesions associates with less severe disease and absence of oligoclonal bands in MS Neurol. Neuroimmunol. Neuroinflamm. 8, e955 (2021).

51. Absinta, M. et al. Gadolinium-based MR characterization of leptomeningeal inflammation in multiple sclerosis. Neurology 85, 18-28 (2015).

52. Magliozzi, R. et al. Meningeal B-cell follicles in secondary progressive multiple sclerosis associate with early onset of disease and severe cortical pathology. Brain 130, 1089-1104 (2007)

53. Cepok, S. et al. Patterns of cerebrospinal fluid pathology correlate with disease progression in multiple sclerosis. Brain 124, 2169-2176 (2001).

54. Magliozzi, R. et al. A Gradient of neuronal loss and meningeal inflammation in multiple sclerosis. Ann. Neurol. 68, 477-493 (2010).

This publication provides strong evidence that follicle-like structures in the meninges are associated with severe cortical pathology not seen in other types of MS lesions.

55. Howell, O. W. et al. Extensive grey matter pathology in the cerebellum in multiple sclerosis is linked to inflammation in the subarachnoid space. Neuropathol. Appl. Neurobiol. 41, 798-813 (2015).

56. Lovato, L. et al. Related B cell clones populate the meninges and parenchyma of patients with multiple sclerosis. Brain 134, 534-541 (2011)

57. Lucchinetti, C. F. et al. Inflammatory cortical demyelination in early multiple sclerosis. N. Engl. J. Med. 365, 2188-2197 (2011)

58. Peterson, J. W., Bo, L., Mork, S., Chang, A. \& Trapp, B. D. Transected neurites, apoptotic neurons, and reduced inflammation in cortical multiple sclerosis lesions. Ann. Neurol. 50, 389-400 (2001).

59. Magliozzi, R. et al. Inflammatory intrathecal profiles and cortical damage in multiple sclerosis. Ann. Neurol. 83, 739-755 (2018)

60. Mitsdorffer, M. et al. Formation and immunomodulatory function of meningeal B-cell aggregates in progressive CNS autoimmunity. Brain 144, 1697-1710 (2021)

61. Pikor, N. B. et al. Integration of Th17- and lymphotoxin-derived signals initiates meningealresident stromal cell remodeling to propagate neuroinflammation. Immunity 43, 1160-1173 (2015)

62. Grewal, I. S. et al. CD62L is required on effector cells for local interactions in the CNS to cause myelin damage in experimental allergic encephalomyelitis. Immunity 14, 291-302 (2001)

63. Dang, A. K., Tesfagiorgis, Y., Jain, R. W., Craig, H. C. $\&$ Kerfoot, S. M. Meningeal infiltration of the spinal cord by non-classically activated B cells is associated with chronic disease course in a spontaneous B cell-dependent model of CNS autoimmune disease. Front. Immunol. 6, 470 (2015)

64. Serafini, B., Rosicarelli, B., Magliozzi, R., Stigliano, E. $\&$ Aloisi, F. Detection of ectopic B-cell follicles with germinal centers in the meninges of patients with secondary progressive multiple sclerosis. Brain Pathol. 14, 164-174 (2004).

65. Michel, L. et al. Activated leukocyte cell adhesion molecule regulates $\mathrm{B}$ lymphocyte migration across central nervous system barriers. Sci. Transl. Med. 11, eaaw0475 (2019)

Using a combination of mouse models and live imaging, this publication identifies ALCAM as an important mediator of B cell recruitment into the CNS and quantifies other recruitment molecules on $B$ cells.

66. Alter, A. et al. Determinants of human B cell migration across brain endothelial cells. J. Immunol. 170, 4497-4505 (2003).

67. Haas, J. et al. The choroid plexus is permissive for a preactivated antigen-experienced memory B-cell subset in multiple sclerosis. Front. Immunol. 11, 618544 (2020).

68. van Langelaar, J. et al. Induction of brain-infiltrating T-bet-expressing B cells in multiple sclerosis. Ann. Neurol. 86, 264-278 (2019).

69. Hussain, R. Z. et al. alpha4-integrin deficiency in $B$ cells does not affect disease in a T-cell-mediated EAE disease model. Neurol. Neuroimmunol. Neuroinflamm 6, e563 (2019).

70. Lehmann-Horn, K., Sagan, S. A., Bernard, C. C. Sobel, R. A. \& Zamvil, S. S. B-cell very late antigen-4 deficiency reduces leukocyte recruitment and susceptibility to central nervous system autoimmunity. Ann. Neurol. 77, 902-908 (2015)

71. Hausler, D. et al. CNS inflammation after natalizumab therapy for multiple sclerosis: A retrospective histopathological and CSF cohort study. Brain Pathol. 31, e12969 (2021)

72. Tesfagiorgis, Y., Zhu, S. L., Jain, R. \& Kerfoot, S. M. Activated $B$ cells participating in the anti-myelin response are excluded from the inflamed central nervous system in a model of autoimmunity that allows for B cell recognition of autoantigen. J. Immunol. 199, 449-457 (2017).

73. Pollok, K. et al. The chronically inflamed central nervous system provides niches for long-lived plasma cells. Acta Neuropathol. Commun. 5, 88 (2017).

74. Rojas, O. L. et al. Recirculating Intestinal IgAproducing cells regulate neuroinflammation via IL-10. Cell 176, 610-624.e18 (2019).

This study identified that IgA ${ }^{+}$plasma cells from the gut move into the CNS during neuroinflammation and suppress EAE using IL-10.

75. Nguyen, D. C. et al. Factors of the bone marrow microniche that support human plasma cell survival and immunoglobulin secretion. Nat. Commun. 9 , 3698 (2018)

76. Baker, D., Pryce, G., James, L. K., Schmierer, K. \& Giovannoni, G. Failed B cell survival factor trials support the importance of memory B cells in multiple sclerosis. Eur. J. Neurol. 27, 221-228 (2020).

77. Touil, H. et al. Human central nervous system astrocytes support survival and activation of B cells: implications for MS pathogenesis. J. Neuroinflammation 15, 114 (2018).

78. Chan, T. D. et al. Antigen affinity controls rapid T-dependent antibody production by driving the expansion rather than the differentiation or extrafollicular migration of early plasmablasts. J. Immunol. 183, 3139-3149 (2009).
79. Quinn, J. L., Kumar, G., Agasing, A., Ko, R. M. \& Axtell, R. C. Role of TFH cells in promoting T helper 17-induced neuroinflammation. Front. Immunol. $\mathbf{9}$, 382 (2018)

80. Chihara, N. et al. Plasmablasts as migratory IgGproducing cells in the pathogenesis of neuromyelitis optica. PLoS One 8, e83036 (2013).

81. O'Connor, T. et al. Age-related gliosis promotes central nervous system lymphoma through CCL19-mediated tumor cell retention. Cancer Cell 36, 250-267.e9 (2019).

82. Williams, M. T. et al. Interleukin-15 enhances cellular proliferation and upregulates CNS homing molecules in pre-B acute lymphoblastic leukemia. Blood 123, 3116-3127 (2014).

83. Krumbholz, M. et al. BAFF is produced by astrocytes and up-regulated in multiple sclerosis lesions and primary central nervous system lymphoma. J. Exp. Med. 201, 195-200 (2005)

84. DiSano, K. D., Royce, D. B., Gilli, F. \& Pachner, A. R. Central nervous system inflammatory aggregates in the Theiler's virus model of progressive multiple sclerosis. Front. Immunol. 10, 1821 (2019).

85. Hohlfeld, R., Dornmair, K., Meinl, E. \& Wekerle, H. The search for the target antigens of multiple sclerosis, part 2: $C D 8^{+} T$ cells, $B$ cells, and antibodies in the focus of reverse-translational research. Lancet Neurol. 15, 317-331 (2016)

86. Lucchinetti, C. et al. Heterogeneity of multiple sclerosis lesions: implications for the pathogenesis of demyelination. Ann. Neurol. 47, 707-717 (2000).

87. Sadaba, M. C. et al. Axonal and oligodendrocytelocalized IgM and IgG deposits in MS lesions. J. Neuroimmunol. 247, 86-94 (2012).

88. Genain, C. P., Cannella, B., Hauser, S. L. \& Raine, C. S. Identification of autoantibodies associated with myelin damage in multiple sclerosis. Nat. Med. 5, 170-175 (1999).

89. Zhang, Y. et al. Clonal expansion of IgA-positive plasma cells and axon-reactive antibodies in MS lesions. J. Neuroimmunol. 167, 120-130 (2005).

90. Keegan, M. et al. Relation between humoral pathological changes in multiple sclerosis and response to therapeutic plasma exchange. Lancet 366, 579-582 (2005)

91. Silber, E., Semra, Y. K., Gregson, N. A. \& Sharief, M. K. Patients with progressive multiple sclerosis have elevated antibodies to neurofilament subunit. Neurology 58, 1372-1381 (2002).

92. Blauth, K. et al. Antibodies produced by clonally expanded plasma cells in multiple sclerosis cerebrospinal fluid cause demyelination of spinal cord explants. Acta Neuropathol. 130, 765-781 (2015).

93. Brandle, S. M. et al. Distinct oligoclonal band antibodies in multiple sclerosis recognize ubiquitous self-proteins. Proc. Natl Acad. Sci. USA 113 7864-7869 (2016).

94. Willis, S. N. et al. Investigating the antigen specificity of multiple sclerosis central nervous system-derived immunoglobulins. Front. Immunol. 6, 600 (2015).

95. Benjamins, J. A et al. Exosome-enriched fractions from MS B cells induce oligodendrocyte death. Neurol. Neuroimmunol. Neuroinflamm. 6, e550 (2019). This paper identified that exosomes produced from $B$ cells can be toxic to oligodendrocytes, opening an entire new field of study.

96. Lisak, R. P. et al. B cells from patients with multiple sclerosis induce cell death via apoptosis in neurons in vitro. J. Neuroimmunol. 309, 88-99 (2017)

97. Bettelli, E., Baeten, D., Jager, A., Sobel, R. A. \& Kuchroo, V. K. Myelin oligodendrocyte glycoproteinspecific $T$ and $B$ cells cooperate to induce a Devic-like disease in mice. J. Clin. Invest. 116, 2393-2402 (2006).

98. Kuerten, S. et al. Tertiary lymphoid organ development coincides with determinant spreading of the myelinspecific T cell response. Acta Neuropathol 124. 861-873 (2012)

99. Parker Harp, C. R. et al. B cells are capable of independently eliciting rapid reactivation of encephalitogenic CD4 T cells in a murine model of multiple sclerosis. PLoS One 13, e0199694 (2018).

100. Pierson, E. R., Stromnes, I. M. \& Goverman, J. M. $B$ cells promote induction of experimental autoimmune encephalomyelitis by facilitating reactivation of $\mathrm{T}$ cells in the central nervous system. J. Immunol. 192 929-939 (2014).

101. Claes, N. et al. Age-associated B cells with proinflammatory characteristics are expanded in a proportion of multiple sclerosis patients. J. Immunol. 197, 4576-4583 (2016) 
102. Li, R. et al. Proinflammatory GM-CSF-producing $B$ cells in multiple sclerosis and B cell depletion therapy. Sci. Transl. Med. 7, 310ra166 (2015). In this study, GM-CSF-producing B cells are shown to induce inflammatory polarization of macrophages, creating a link between B cell and macrophage biology in MS.

103. McWilliam, O., Sellebjerg, F., Marquart, H. V. $\delta$ von Essen, M. R. B cells from patients with multiple sclerosis have a pathogenic phenotype and increased $\mathrm{LT} \alpha$ and TGF $\beta 1$ response. J. Neuroimmunol. 324, 157-164 (2018).

104. Stein, J. et al. Intrathecal B cells in ms have significantly greater lymphangiogenic potential compared to B cells derived from non-MS subjects. Front. Neurol. 9, 554 (2018).

105. Gardner, C. et al. Cortical grey matter demyelination can be induced by elevated pro-inflammatory cytokines in the subarachnoid space of MOG immunized rats. Brain 136, 3596-3608 (2013).

106. Magliozzi, R. et al. Meningeal inflammation changes the balance of TNF signalling in cortical grey matter in multiple sclerosis. J. Neuroinflammation 16, 259 (2019).

107. James, R. E. et al. Persistent elevation of intrathecal pro-inflammatory cytokines leads to multiple sclerosislike cortical demyelination and neurodegeneration. Acta Neuropathol. Commun. 8, 66 (2020).

108. van Olst, L. et al. Meningeal inflammation in multiple sclerosis induces phenotypic changes in cortical microglia that differentially associate with neurodegeneration. Acta Neuropathol. 141, 881-899 (2021).

109. Fehres, C. M. et al. APRIL induces a novel subset of $\operatorname{lgA}^{+}$regulatory $B$ cells that suppress inflammation via expression of IL-10 and PD-L1. Front. Immunol. 10, 1368 (2019).

110. Fillatreau, S., Sweenie, C. H., McGeachy, M. J., Gray, D. \& Anderton, S. M. B cells regulate autoimmunity by provision of IL-10. Nat. Immunol. 3, 944-950 (2002)

111. Pennati, A et al. Regulatory B cells induce formation of IL-10-expressing T cells in mice with autoimmune neuroinflammation. J. Neurosci. 36, 12598-12610 (2016).
112. Bjarnadottir, K. et al. B cell-derived transforming growth factor-beta 1 expression limits the induction phase of autoimmune neuroinflammation. Sci. Rep. 6 , 34594 (2016)

113. Piancone, F. et al. B lymphocytes in multiple sclerosis: $B_{\text {regs }}$ and BTLA/CD272 expressing-CD19+ lymphocytes modulate disease severity. Sci. Rep. 6, 29699 (2016).

114. Shen, P. et al. IL-35-producing B cells are critical regulators of immunity during autoimmune and infectious diseases. Nature 507, 366-370 (2014).

115. Khan, A. R. et al. PD-L1 hi B cells are critical regulators of humoral immunity. Nat. Commun. 6, 5997 (2015).

116. Lehmann-Horn, K. et al. CNS accumulation of regulatory B cells is VLA-4-dependent. Neurol. Neuroimmunol. Neuroinflamm. 3, e21 2 (2016)

117. Matsumoto, M. et al. Interleukin-10-producing plasmablasts exert regulatory function in autoimmune inflammation. Immunity 41, 1040-1051 (2014). This study demonstrates that plasmablasts are a major regulatory population in EAE induced through immunization that inhibits $\mathrm{T}$ cell responses in the lymph nodes where $T$ cells are differentiating

118. Wang, A., Rojas, O., Lee, D. \& Gommerman, J. L. Regulation of neuroinflammation by $B$ cells and plasma cells. Immunol. Rev. 299, 45-60 (2021).

119. Stebegg, M. et al. Regulation of the germinal center response. Front. Immunol. 9, 2469 (2018).

120. Roco, J. A. et al. Class-switch recombination occurs infrequently in germinal centers. Immunity $\mathbf{5 1}$, 337-350.e7 (2019).

121. Lepennetier, G. et al. Cytokine and immune cell profiling in the cerebrospinal fluid of patients with neuro-inflammatory diseases. J. Neuroinflammation 16, 219 (2019).

122. Flach, A. C. et al. Autoantibody-boosted T-cell reactivation in the target organ triggers manifestation of autoimmune CNS disease. Proc. Natl Acad. Sci. USA 113, 3323-3328 (2016).

123. Romanelli, E. et al. Myelinosome formation represents an early stage of oligodendrocyte damage in multiple sclerosis and its animal model. Nat. Commun. 7 13275 (2016)

124. Jegou, J. F. et al. C3d binding to the myelin oligodendrocyte glycoprotein results in an exacerbated experimental autoimmune encephalomyelitis. J. Immunol 178, 3323-3331 (2007)

125. Molnarfi, N. et al. MHC class II-dependent B cell APC function is required for induction of CNS autoimmunity independent of myelin-specific antibodies. J. Exp. Med. 210, 2921-2937 (2013).

126. Murakami, M., Kamimura, D. \& Hirano, T. Pleiotropy and specificity: insights from the interleukin 6 family of cytokines. Immunity 50, 812-831 (2019).

127. Pennati, A., Nylen, E. A., Duncan, I. D. \& Galipeau, J. Regulatory B cells normalize CNS myeloid cell content in a mouse model of multiple sclerosis and promote oligodendrogenesis and remyelination. J. Neurosci. 40, 5105-5115 (2020)

Acknowledgements

R.W.J. acknowledges postdoctoral fellowship funding from the University of Calgary Eyes High program, the Multiple Sclerosis of Canada and Roche unrestricted educational fellowship. V.W.Y. acknowledges salary support from the Canada Research Chair Tier 1 program and operating grant support from the Canadian Institutes of Health Research and the Multiple Sclerosis Society of Canada.

Author contributions

The authors contributed equally to all aspects of the article.

\section{Competing interests}

The authors declare no competing interests.

Peer review information

Nature Reviews Immunology thanks S. Pillai and the other, anonymous, reviewer(s) for their contribution to the peer review of this work.

\section{Publisher's note}

Springer Nature remains neutral with regard to jurisdictional claims in published maps and institutional affiliations.

\section{Supplementary information}

The online version contains supplementary material available at https://doi.org/10.1038/s41577-021-00652-6.

(C) Springer Nature Limited 2021 